\title{
Política Nacional de Saúde Integral da População Negra: implementação, conhecimento e aspectos socioeconômicos sob a perspectiva desse segmento populacional
}

\author{
The National Comprehensive Health Policy for the Black \\ Population: implementation, awareness and socioeconomic aspects \\ from the perspective of this ethnic group
}

José Antônio Chehuen Neto ${ }^{1}$

Geovane Mostaro Fonseca ${ }^{1}$

Igor Vilela Brum ${ }^{1}$

João Luís Carvalho Tricote dos Santos ${ }^{1}$

Tamara Cristina Gomes Ferraz Rodrigues ${ }^{1}$

Katia Rocha Paulino ${ }^{1}$

Renato Erothildes Ferreira ${ }^{1}$

${ }^{1}$ Faculdade de Medicina, Universidade Federal de Juiz de Fora. Av. Eugênio do Nascimento $s / n^{\circ}$, Dom Bosco. 36038-330 Juiz de Fora MG Brasil. chehuen.neto@ yahoo.com.br
Abstract The scope of the National Comprehensive Health Policy for the Black Population is to ensure equitable health care to this population. This policy is to compensate for the discrimination suffered by this ethnic group throughout the history of Brazil. The black population presents higher social and economic vulnerability, leading to shorter life expectancy and an increased susceptibility to diseases. The objective of the study is to investigate the black population's knowledge about this policy, its potential benefits and the difficulties of this population regarding access to health. It involves cross-sectional, quantitative and descriptive research. Structured interviews were conducted with 391 black people in Juiz de Fora. The sample was structured according to race (black and brown). Around 90\% of the sample reported not knowing the existence of a health policy for the black population, and 53\% stated that this policy could enhance racial discrimination. Having completed primary education and lower income was positively associated with higher discrimination in health care. Most of the sample didn't know the existence of the PNSIPN, but was in favor of its goals, despite the possibility that it can reinforce the legacy of Brazilian racial discrimination.

Key words Public health, African continental ancestry group, Health policy, Social discrimination, Public opinion
Resumo A Política Nacional de Saúde Integral da População Negra tem o objetivo de garantir a equidade na atenção à saúde para esse segmento populacional. Tal medida possui um caráter compensatório em virtude das discriminações raciais existentes ao longo da história do Brasil. A população negra apresenta maior vulnerabilidade social e econômica, o que reflete uma menor expectativa de vida e maior susceptibilidade a agravos. O objetivo do estudo é investigar o conhecimento da população negra acerca da política, seus potenciais benefícios e as dificuldades de acesso à saúde. Trata-se de uma pesquisa transversal, descritiva e quantitativa. Foram realizadas entrevistas estruturadas com 391 indivíduos negros, usuários do SUS, da cidade de Juiz de Fora. A amostra foi estratificada de acordo com raça (preto e pardo), renda e escolaridade. Cerca de 90\% dos entrevistados relataram desconhecer a existência de uma política de saúde para a população negra e 53\% declararam uma possível discriminação racial. Observou-se também associação positiva entre discriminação e menor escolaridade e renda. Apesar de desconhecerem a existência da PNSIPN, a maioria dos entrevistados aprovou seus objetivos, mesmo relatando a possibilidade de discriminação dela advinda.

Palavras-chave Saúde pública, Grupo com ancestrais do continente africano, Politica de saúde, Discriminação social, Opinião pública 


\section{Introdução}

As políticas públicas dos últimos anos, direcionadas às pessoas em condições de vulnerabilidade, contribuíram para a redução das desigualdades no Brasil. Em se tratando da saúde, a redução das desigualdades tem ocorrido por uma série de políticas de promoção da equidade, desenvolvidas pelo Ministério da Saúde. Uma delas é a Política Nacional de Saúde Integral da População Negra (PNSIPN), cujo objetivo é "garantir a equidade na efetivação do direito humano à saúde da população negra em seus aspectos de promoção, prevenção, atenção, tratamento e recuperação de doenças e agravos transmissíveis e não transmissíveis, incluindo aqueles de maior prevalência nesse segmento populacional, como por exemplo, a doença falciforme, miomatose, diabetes tipo 2, dentre outras"1-3.

Na década de 80, teve início no Brasil uma série de movimentos sociais negros em busca de melhores condições de vida. Um deles foi a "Marcha Zumbi dos Palmares", em 1995, que preconizava o fim do racismo, melhores condições de vida à população negra e fim das desigualdades raciais. Motivada por tais movimentos sociais, em busca de melhores condições de saúde e da maior equidade no Sistema Único da Saúde (SUS) ${ }^{4}$, a PNSIPN foi aprovada em 2007, no dia nacional da Consciência Negra, 20 de novembro, pelo Conselho Nacional de Saúde 1 .

Em linhas gerais, a PNSIPN surge como uma medida compensatória na tentativa de minimizar os efeitos da discriminação e da exploração sofridas pelos negros ao longo da história brasileira, já que esse histórico se reflete em vários aspectos, não sendo diferente em relação à saúde. Desse modo, o reconhecimento social e político ocorre em concordância com a análise dos aspectos de vida e saúde da população negra, sendo atrelados à possibilidade de benefícios decorrentes da execução de uma política de saúde organizada ${ }^{3}$.

De acordo com o censo demográfico do Instituto Brasileiro de Geografia e Estatística (IBGE) de $2010,47,7 \%$ da população brasileira se autodeclara branca, 50,7\%, negra (pretos e pardos) e $1,5 \%$, indígenas e amarelos, indicando, além da prevalência de afrodescendentes na composição étnica do país, um maior reconhecimento do perfil étnico-racial por parte da população brasileira ${ }^{5}$. Em relação aos aspectos socioeconômicos, os dados desse censo revelam que, enquanto o percentual de analfabetos entre os brancos com 15 anos ou mais era de 5,9\%, entre os negros esse valor atingiu $27,4 \%$. Outro exemplo é que bran- cos têm renda $84,5 \%$ maior que pretos e $81,95 \%$ maior que pardos ${ }^{6}$. A implementação efetiva de políticas específicas nesse âmbito, como a PNSIPN, emerge, então, como uma ferramenta importante na reestruturação da saúde através de uma política que adere à demanda e à realidade do país ${ }^{7}$.

Diante dessa realidade, é importante determinar se as desigualdades em saúde estão relacionadas à raça como fator independente ou apenas são consequências dos piores indicadores socioeconômicos apresentados pela população negra ${ }^{8}$.

O Relatório Anual das Desigualdades Raciais no Brasil $^{9}$ mostra a diminuição da qualidade e da expectativa de vida da população negra, e revela maiores chances de morte materna e infantil e menor acesso a serviços de saúde, além da violência vivenciada de forma mais intensa por esse grupo, sobretudo entre jovens negros, quando comparada ao restante da população. Diante das desvantagens materiais e simbólicas historicamente sofridas pelos negros ${ }^{5}$, a escassez de ações efetivas para o seu enfrentamento poderá prorrogar as disparidades nas condições de vida e saúde indefinidamente 9 .

A dificuldade de obter e de agrupar informações sobre a realidade da saúde dos negros no país pode se justificar, em parte, pelos ideais de que no Brasil inexistiam obstáculos de ordem estrutural, social, cultural ou racial para a construção de uma nação verdadeiramente democrática. Esse pensamento, expresso nas obras de intelectuais do século 20, como Gilberto Freyre ${ }^{10}$ e Sérgio Buarque de Holanda"1 ${ }^{11}$ deu origem ao "Mito da Democracia Racial", segundo o qual haveria no Brasil uma convivência pacífica entre as etnias e que todos teriam chances iguais individualmente de sucesso. Com a intenção de reverter o histórico de enigmas em relação à abordagem e investigação de temas relacionados à desigualdade racial, é preciso cada vez mais inquirir as possíveis singularidades quanto à saúde deste grupo étnico no nosso país.

Desse modo, a inclusão do item "identificação racial” em estudos científicos mostrar-se-ia fundamental para uma melhor compreensão das condições políticas, sociais, econômicas e suas consequências no processo saúde-doença da população negra.

Neste estudo, investigamos o conhecimento e a opinião da população negra acerca dessa política recém-implantada no Brasil, os seus potenciais benefícios, as percepções quanto às dificuldades de acesso e de discriminação no que se refere à assistência em saúde. 


\section{Metodologia}

O delineamento do estudo é do tipo transversal, exploratório e descritivo, com características quantitativas ${ }^{12}$. Trata-se de uma pesquisa aplicada, original, realizada na cidade de Juiz de Fora, Minas Gerais, nas suas cinco regiões demográficas mais densamente povoadas ${ }^{6}$, com o objetivo de facilitar a coleta de dados (norte, sul, centro, leste e oeste).

O tamanho da amostra, calculado para a investigação em desfechos de saúde, foi de 391 indivíduos, cujo espectro amostral atende rigorosamente aos critérios e às necessidades estatísticas, com erro amostral de 4,5\% (para mais ou para menos).

Os critérios de inclusão foram: declarar-se da cor preta ou parda, ser morador da cidade de Juiz de Fora/MG, usuário do SUS e ter idade $\geq$ 18 anos, sendo a amostra admitida por conveniência. Como perda amostral foram considerados os questionários interrompidos por qualquer motivo, dados incompletos e a não devolução do Termo de Consentimento Livre e Esclarecido (TCLE) assinado.

Para o levantamento de informações foi utilizada a técnica de entrevista, tendo por base um questionário estruturado composto por 06 questões de cunho socioeconômico e mais 16 questões dicotômicas, ou seja, que admitiam apenas duas respostas mutuamente exclusivas (sim ou não) acerca da Política Nacional de Saúde Integral da População Negra, acompanhada do TCLE em duas vias.

Os participantes foram abordados de forma padronizada por pesquisador treinado, receberam informações básicas sobre o estudo e, ao serem convidados a participar, consentiram voluntariamente nessa participação com a assinatura do TCLE. Foram três responsáveis pela coleta de dados, participantes da Disciplina de Metodologia Científica na Saúde do curso médico.

As variáveis investigadas foram divididas em dois grupos: quantitativas contínuas (apenas para a idade) e qualitativas dicotômicas. A análise, descritiva e exploratória, utilizou frequências absolutas (n), frequências relativas (\%), medidas de tendência central (média) e medidas de dispersão (desvio-padrão).

Para a análise comparativa das proporções das variáveis dicotômicas (associação entre estas variáveis), foi aplicado o Teste do Qui-Quadrado de Independência (sem correção). O nível de significância para este teste foi $\mathrm{p}$-valor $\leq 0,05$ para um intervalo de confiança de $95 \%$.
Para uma variável dependente que assume apenas dois valores, como em nossa pesquisa, o crosstabs foi a estratégia analítica escolhida para a estimação do risco de insucesso associado às diversas variáveis consideradas. Como usual, apresentamos os resultados a partir da estimação do Odds Ratio (OR).

A pesquisa foi desenvolvida nos meses seguintes após a aprovação do projeto pelo Comitê de Ética em Pesquisa da Universidade Federal de Juiz de Fora (CEP/UFJF). A participação na pesquisa implicou risco mínimo aos participantes, ou seja, não houve interferência do pesquisador em nenhum aspecto do bem-estar físico, psicológico e social, bem como da intimidade, conforme os parâmetros contidos na Resolução 466/12 ${ }^{13}$ do Conselho Nacional de Saúde/Ministério da Saúde, que dispõe sobre pesquisas envolvendo seres humanos.

O protocolo foi aprovado pelo CEP/UFJF. Para o tratamento estatístico e montagem do banco de dados foi utilizado o Software estatístico SPSS Versão $15.0^{\circledR}, 2010$.

\section{Resultados}

Para obtenção da amostra, foram abordadas 523 pessoas, sendo que 123 se recusaram a participar da entrevista (23,5\%). Dos 400 entrevistados, 9 se enquadraram nos critérios de perda amostral $(2,25 \%)$, contemplando uma amostra de 391 indivíduos.

Visando facilitar a análise estatística, os dados foram organizados tematicamente, de forma a destacar os indicadores sociais considerados mais relevantes. Esses indicadores incluem: sexo feminino ou masculino, se declarar preto ou pardo, ganhar até 03 salários mínimos ou acima, e ter cursado até o ensino fundamental ou escolaridade superior a esta.

A população avaliada é relativamente jovem, com média de idade de $36,7 \pm 17,2$ anos e mediana de 34 anos. A distribuição dos indivíduos avaliados conforme o gênero apresenta cerca de $54,5 \%$ de mulheres e $45,5 \%$ de homens. Com relação à raça/cor declarada pelos indivíduos, a amostra possui predominância de pretos com $62,1 \%$, contra $37,9 \%$ de pardos. Dentre os entrevistados, $61,0 \%$ estudaram além do $9^{\circ}$ ano do ensino fundamental. Em relação à renda, 69,1\% possuem renda menor do que 03 salários mínimos (Tabela 1).

Quando avaliado o conhecimento sobre a existência de uma política de saúde pública es- 
pecífica para a população negra, 90,5\% dos entrevistados relataram desconhecer qualquer ação nesse sentido. No que se refere à necessidade de políticas de saúde com essa finalidade, $60,9 \%$ da amostra afirmaram sua importância. Entretanto, $52,7 \%$ declararam que a PNSIPN tende a reforçar a discriminação racial.

Em relação às diferenças entre pretos e pardos, $41,6 \%$ dos pretos e $54,7 \%$ dos pardos relataram que não enfrentam dificuldades financeiras nas despesas de saúde $(\mathrm{OR}=25 \%)$. Os pretos declararam não saber onde procurar atendimento médico com frequência cerca de duas vezes maior que os pardos $(\mathrm{OR}=90 \%)$. Quando inda-

Tabela 1. Percentuais das condições socioeconômicas.

\begin{tabular}{lcc}
\hline \multirow{2}{*}{ Item } & \multicolumn{2}{c}{ Distribuição } \\
\cline { 2 - 3 } & $\mathrm{N}$ & $\%$ \\
\hline Idade & & \\
$\quad \leq 34$ anos & 200 & 51,2 \\
$\quad>34$ anos & 191 & 48,8 \\
Cor & & \\
$\quad$ Preto & 243 & 62,1 \\
$\quad$ Pardo & 148 & 37,9 \\
Renda & & \\
$\quad$ Até 03 salários mínimos & 270 & 69,1 \\
$\quad>$ de 03 salários mínimos & 121 & 30,9 \\
Escolaridade & & \\
$\quad$ Até o 9o ano do fundamental & 153 & 39,0 \\
$\quad>$ do 90 ano do fundamental & 238 & 61,0 \\
Sexo & & \\
$\quad$ Feminino & 213 & 54,5 \\
$\quad$ Masculino & 178 & 45,5 \\
\hline
\end{tabular}

gados sobre o acesso da população negra à saúde, tem-se que o preto considerou tal acesso mais difícil $(\mathrm{OR}=20 \%)$. Os pretos, com maior frequência, julgaram que os negros (incluindo pardos e pretos) se preocupam menos com a saúde (OR $=18 \%)$ (Tabela 2$)$.

Em relação à renda, diferenças importantes foram observadas na comparação dos dois estratos. Os indivíduos com renda superior a 03 salários mínimos apresentaram maior insatisfação com o serviço público de saúde $(\mathrm{OR}=14 \%)$ e menor dificuldade com despesas de saúde (OR $=70 \%)$. Quanto à discriminação 48,5\% dos que possuem renda inferior a 03 salários mínimos e $38,8 \%$ dos que têm renda superior a essa consideraram a ocorrência de discriminação no atendimento à saúde $(\mathrm{OR}=80 \%)$. Quando questionados se já sofreram discriminação no atendimento à saúde, os indivíduos de baixa renda responderam que sim com frequência significativamente maior, $9,3 \%$ contra $2,5 \%(\mathrm{OR}=270 \%)$. Além disso, os indivíduos com renda acima de 03 salários mínimos mostraram maior conhecimento sobre a existência de doenças mais prevalentes na população negra $(\mathrm{OR}=40 \%)$. Os indivíduos com menor renda declararam com maior frequência que o negro tem maior propensão à morte violenta. Os entrevistados que ganhavam até 03 salários mínimos consideraram com maior frequência que os entrevistados com renda superior $(\mathrm{OR}=20 \%)$ que a política reforça a discriminação racial (Tabela 3 ).

As diferenças de opinião entre os entrevistados com maior e menor escolaridade revelaram que aqueles com escolaridade até o ensino fundamental completo estão mais satisfeitos com os

\begin{tabular}{|c|c|c|c|c|c|c|c|c|}
\hline \multirow[b]{2}{*}{ Questão } & \multirow[b]{2}{*}{ Categoria } & \multicolumn{2}{|c|}{ Sim } & \multicolumn{2}{|c|}{ Não } & \multirow[b]{2}{*}{ Sig. } & \multirow[b]{2}{*}{ OR } & \multirow[b]{2}{*}{ IC 95\% } \\
\hline & & $\mathbf{N}$ & $\%$ & $\mathrm{~N}$ & $\%$ & & & \\
\hline \multirow[t]{2}{*}{04} & Preto & 142 & 58,4 & 101 & 41,6 & \multirow[t]{2}{*}{0,01} & \multirow[t]{2}{*}{$25,0 \downarrow$} & \multirow[t]{2}{*}{$0,61-0,93$} \\
\hline & Pardo & 67 & 45,3 & 81 & 54,7 & & & \\
\hline \multirow[t]{2}{*}{05} & Preto & 203 & 83,5 & 40 & 16,5 & \multirow[t]{2}{*}{0,03} & \multirow[t]{2}{*}{$90,0 \uparrow$} & \multirow[t]{2}{*}{$1,03-2,38$} \\
\hline & Pardo & 135 & 91,2 & 13 & 8,8 & & & \\
\hline \multirow[t]{2}{*}{09} & Preto & 137 & 56,4 & 106 & 43,6 & \multirow[t]{2}{*}{0,04} & \multirow[t]{2}{*}{$20,0 \downarrow$} & \multirow[t]{2}{*}{$0,65-0,99$} \\
\hline & Pardo & 68 & 45,9 & 80 & 54,1 & & & \\
\hline \multirow[t]{2}{*}{10} & Preto & 100 & 41,2 & 143 & 58,8 & \multirow[t]{2}{*}{0,01} & \multirow[t]{2}{*}{$18,0 \downarrow$} & \multirow[t]{2}{*}{$0,71-0,96$} \\
\hline & Pardo & 43 & 29,1 & 105 & 70,9 & & & \\
\hline
\end{tabular}

Nota 1: Os dados das linhas representam o total da amostra (100\%) em cada categoria. Nota 2 OR (Odds Ratio): Valores diferentes de (1) foram convertidos em porcentagem. Valores de OR e IC na linha superior de cada questão representam a diferença de prevalência de SIM, e na linha inferior, de NÃO, sendo representado o de maior valor. Se $\uparrow$, é fator de risco, se $\downarrow$, é fator de proteção. Nota 3: Sig. (Significância estatística das diferenças) teste de $\chi^{2}$ de Pearson. Questão 04: As despesas de saúde são um problema? Questão 05: Sabe onde procurar atendimento médico? Questão 09: Você acha o acesso à saúde mais difícil para população negra? Questão 10: Você acha a população negra menos preocupada com a saúde? 


\begin{tabular}{|c|c|c|c|c|c|c|c|c|}
\hline \multirow[b]{2}{*}{ Questão } & \multirow[b]{2}{*}{ Categoria } & \multicolumn{2}{|c|}{ Sim } & \multicolumn{2}{|c|}{ Não } & \multirow[b]{2}{*}{ Sig. } & \multirow[b]{2}{*}{ OR } & \multirow[b]{2}{*}{ IC 95\% } \\
\hline & & $\mathbf{N}$ & $\%$ & $\mathbf{N}$ & $\%$ & & & \\
\hline \multirow[t]{2}{*}{02} & $\leq 3$ salários & 96 & 35,6 & 174 & 64,4 & 0,05 & $14,0 \downarrow$ & $0,15-0,99$ \\
\hline & > 3 salários & 31 & 25,6 & 90 & 74,4 & & $70,0 \uparrow$ & $1,30-2,16$ \\
\hline \multirow[t]{2}{*}{04} & $\leq 3$ salários & 165 & 61,1 & 105 & 38,9 & 0,00 & & \\
\hline & > 3 salários & 44 & 36,4 & 77 & 63,6 & & $80,0 \downarrow$ & $0,08-0,53$ \\
\hline \multirow[t]{2}{*}{06} & $\leq 3$ salários & 131 & 48,5 & 139 & 51,5 & 0,04 & & \\
\hline & $>3$ salários & 47 & 38,8 & 74 & 61,2 & & $270,0 \uparrow$ & $1,15-12,1$ \\
\hline \multirow[t]{2}{*}{07} & $\leq 3$ salários & 25 & 9,3 & 245 & 90,7 & 0,01 & & \\
\hline & > 3 salários & 3 & 2,5 & 118 & 97,5 & & & \\
\hline \multirow[t]{2}{*}{08} & $\leq 3$ salários & 110 & 40,7 & 160 & 59,3 & 0,00 & $40,0 \uparrow$ & $1,10-1,73$ \\
\hline & > 3 salários & 69 & 57,0 & 52 & 43,0 & & & \\
\hline \multirow[t]{2}{*}{12} & $\leq 3$ salários & 215 & 79,6 & 55 & 20,4 & 0,00 & $40,0 \downarrow$ & $0,42-0,84$ \\
\hline & > 3 salários & 80 & 66,1 & 41 & 33,9 & & & \\
\hline \multirow[t]{2}{*}{15} & $\leq 3$ salários & 152 & 56,3 & 118 & 43,7 & 0,03 & $21,0 \downarrow$ & $0,64-0,97$ \\
\hline & > 3 salários & 54 & 44,6 & 67 & 55,4 & & & \\
\hline
\end{tabular}

Nota 1: Os dados das linhas representam o total da amostra (100\%) em cada categoria. Nota 2 OR (Odds Ratio): Valores diferentes de (1) foram convertidos em porcentagem. Valores de OR e IC na linha superior de cada questão representam a diferença de prevalência de SIM, e na linha inferior, de NÃO, sendo representado o de maior valor. Se $\uparrow$, é fator de risco, se $\downarrow$, é fator de proteção. Nota 3: Sig. (Significância estatística das diferenças) teste de $\chi^{2}$ de Pearson. Questão 02: Satisfação com o serviço público de saúde (sim)/(não) Questão 04: As despesas de saúde são um problema? Questão 06: Existe discriminação no atendimento em saúde pública? Questão 07: Já sofreu discriminação no SUS? Questão 08: Existem doenças típicas da população negra? Questão 12: O negro tem maior propensão à morte violenta? Questão15: A política reforça a discriminação racial?

serviços de saúde $(\mathrm{OR}=70 \%)$. Em se tratando das dificuldades financeiras, os indivíduos com menor escolaridade relataram maiores dificuldades no acesso e nas despesas com saúde (OR = $70 \%$ e RR $=50 \%$, respectivamente). Para $54,9 \%$ dos entrevistados com menor escolaridade, existe discriminação no atendimento à saúde, contra $39,5 \%$ dos mais escolarizados $(\mathrm{OR}=40 \%)$. Além disso, os entrevistados com menor escolaridade apontaram ter sofrido discriminação com frequência bem maior, $14,4 \%$ contra 2,5\% (OR = $470 \%)$. Observou-se também uma associação positiva entre escolaridade e conhecimento sobre a existência de doenças mais prevalentes $(\mathrm{OR}=$ $22 \%)$. No que se refere ao acesso à saúde, aqueles com maior escolaridade julgaram que o negro não enfrenta maiores dificuldades quando comparados ao restante da população $(\mathrm{OR}=50 \%)$. Por fim, 53,7\% das pessoas com menor escolaridade interpretaram que o negro tem menor preocupação com a saúde, contra 25,6\% dos demais $(\mathrm{OR}=110 \%)($ Tabela 4$)$.

Em relação ao gênero, apenas uma questão apresentou diferença estatisticamente significativa. Observou-se que a parcela masculina da amostra constatou que a população negra tem menor preocupação com a saúde com menor frequência $(\mathrm{OR}=17 \%)$.

\section{Discussão}

A percepção do usuário é de extrema importância na avaliação das ações que vêm sendo desenvolvidas no setor saúde e serve como vetor de direcionamento e planejamento do serviço. Estudo recente sobre a satisfação dos usuários do SUS ${ }^{14}$ mostrou, por exemplo, que $72,0 \%$ dos seus usuários têm suas necessidades resolvidas pelos serviços de saúde. Nossa pesquisa evidenciou que $32,4 \%$ da amostra declarou-se satisfeita com o serviço prestado, considerando que os serviços atendem às necessidades na maioria das vezes, não apontando diferenças significativas entre pretos e pardos. Entretanto, indivíduos com renda acima de 03 salários mínimos mostraram maior insatisfação se comparados àqueles com renda inferior.

O preconceito e a discriminação são variáveis ainda presentes na sociedade brasileira e que afetam os serviços de saúde ${ }^{15}$. Estudo realizado em São Paulo em $2007^{15}$ evidenciou que 60,0\% dos pretos e $40,8 \%$ dos pardos percebem discriminação racial nos serviços de saúde. Entretanto, uma pesquisa realizada pela Fundação Perseu $\mathrm{Abramo}^{16}$, com a população em geral, mostra que $6,0 \%$ dos pretos e 3,0\% dos pardos já sofreram discriminação no atendimento à saúde. 


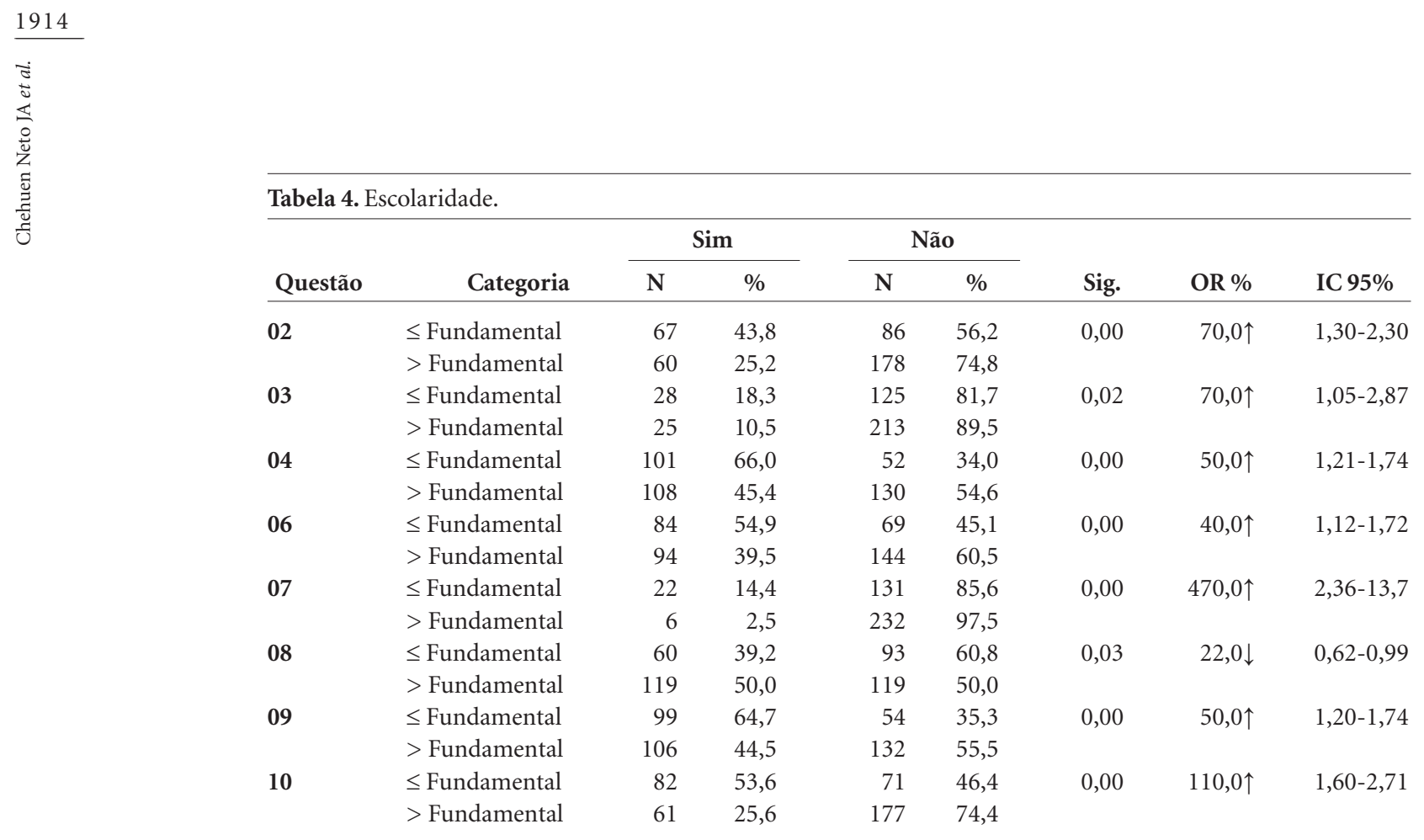

Nota 1: Os dados das linhas representam o total da amostra (100\%) em cada categoria. Nota 2 OR (Odds Ratio): Valores diferentes de (1) foram convertidos em porcentagem. Valores de OR e IC na linha superior de cada questão representam a diferença de prevalência de SIM, e na linha inferior de NÃO, sendo representado o de maior valor. Se $\uparrow$, é fator de risco, se $\downarrow$, é fator de proteção Nota 3: Sig. (Significância estatística das diferenças) teste de $\chi^{2}$ de Pearson. Questão 02: Satisfação com o serviço público de saúde $(\operatorname{sim}) /($ não). Questão 03: Deixou de procurar atendimento médico por dificuldades financeiras? Questão 04: As despesas de saúde são um problema? Questão 06: Existe discriminação no atendimento em saúde pública? Questão 07: Já sofreu discriminação no SUS? Questão 08: Existem doenças típicas da população negra? Questão 09: Você acha o acesso à saúde mais difícil para população negra? Questão 10: Você acha a população negra menos preocupada com a saúde?

Nosso estudo encontrou prevalências semelhantes: $48,6 \%$ dos pretos e $40,5 \%$ dos pardos perceberam discriminação, enquanto que $7,0 \%$ dos pretos e $6,1 \%$ dos pardos relataram já a terem sofrido. Esses resultados sugerem uma discrepância entre a percepção e a ocorrência de fato da discriminação. Uma hipótese seria a dificuldade das pessoas em reconhecer e relatar a própria discriminação. Através da análise dos dados, observou-se também uma associação positiva entre discriminação e idade e uma associação negativa com renda e escolaridade.

Em relação à preocupação da população negra com a saúde, a maioria dos entrevistados negou que esse segmento populacional tenha menor preocupação; no entanto, o preto, com uma prevalência maior do que o pardo, considerou o negro menos preocupado com a saúde. Isso indica que, apesar de a maioria da amostra considerar o acesso do negro à saúde mais difícil, isso não reflete em um abandono e descaso com a saúde.

A intensa miscigenação ocorrida no território brasileiro, incluindo o negro africano, o branco europeu e os indígenas nativos, determinou doen- ças de caráter genético que se manifestam de maneira singular no país ${ }^{17}$. Pode-se considerar que algumas doenças afetam mais a população negra devido tanto a fatores genéticos quanto a fatores socioeconômicos desfavoráveis ${ }^{18}$. Quando indagados sobre o conhecimento de doenças mais prevalentes nessa população, notou-se que pessoas com renda e escolaridade menores desconhecem com maior frequência a existência de doenças mais prevalentes na etnia negra. Essa realidade evidencia a importância de uma divulgação mais ampla acerca dessas particularidades, a fim de reduzir as consequências em saúde decorrentes das desigualdades socioeconômicas. Ainda no que se refere à informação, percebeu-se que os pretos com uma frequência superior aos pardos já deixaram de procurar atendimento médico por não saber onde encontrá-lo, reforçando, novamente, a necessidade de ampliar o acesso à informação.

Um achado interessante do estudo foi que, embora $86,4 \%$ da amostra não tenha relatado dificuldades financeiras na procura de atendimento médico, a maioria dos entrevistados revelou que as despesas com saúde, como procedimentos e medicações, são um problema no orçamento 
familiar. Isso revela a importância de medidas que, além do atendimento, visem facilitar o tratamento do paciente de maneira continuada.

No Brasil, 39 mil negros são assassinados por ano, enquanto esse número se encontra na faixa de 16 mil para as outras raças. Enquanto a expectativa de vida de um indivíduo não negro se reduz em 2,57 anos devido à violência, no indivíduo negro, esta se reduz em 3,5 anos ${ }^{19}$. A maior exposição a substâncias tóxicas em ambientes menos saudáveis, a assistência à saúde inadequada ou degradante e as experiências diretas de atos ou atitudes de discriminação seriam meios de justificar essas desigualdades étnico-sociais na saúde ${ }^{20}$. Dos entrevistados, 75,4\% relataram perceber essa realidade. Nesse cenário, uma diferença observada foi que os indivíduos com renda mais elevada correlacionam menos frequentemente a maior propensão do negro à morte violenta. Essa situação pode ser explicada, uma vez que pessoas com maior renda podem estar menos inseridas em ambientes violentos, o que possivelmente implica em uma menor percepção da mesma.

O presente estudo revelou que, apesar de haver uma política de saúde voltada especificamente à população negra, $90,5 \%$ da amostra entrevistada não a conhece. Isso reflete a falta de informação acerca das políticas públicas de saúde. Nos casos em que os entrevistados relataram desconhecimento da política, eles foram informados pelo entrevistador acerca da mesma. Posteriormente, 60,9\% da amostra considerou a PNSIPN necessária. Em contrapartida, um estudo realizado no estado do Rio de Janeiro ${ }^{21}$ constata que a maioria dos profissionais da atenção primária é contra tal política, pois acreditam que a população negra não apresenta desigualdades que justifiquem uma política especial. De acordo com os entrevistados, a PNSIPN fere o princípio de igualdade prezado pelo SUS.

Apesar de julgarem-na necessária, a maioria dos entrevistados revelou que essa política tende a reforçar a discriminação racial no país. O Mito da Democracia Racial ${ }^{11}$ pode explicar, em partes, os relatos de que uma política específica para a população negra seria uma forma de privilégio e, portanto, desnecessária, uma vez que considera todos os indivíduos iguais independente da raça.

Em linhas gerais, as evidências levantadas no presente estudo sugerem que o ciclo da PNSIPN está incompleto. Desse modo, essa temática deve ser abordada em novas pesquisas com o intuito de avaliar a efetividade e gerar subsídios para o aperfeiçoamento de políticas semelhantes.

\section{Conclusão}

Uma grande parcela da amostra demonstrou-se insatisfeita com o acesso e a qualidade dos serviços prestados na rede pública de saúde em nossa região. Embora não tenham dificuldade no acesso, os custos com o prosseguimento e a continuidade do tratamento médico foram apontados como um dos maiores empecilhos.

Apesar de desconhecerem a existência da PNSIPN, quando apresentados os seus objetivos, a maioria dos entrevistados mostrou-se favorável à mesma. Entretanto, consideram que ela pode reforçar o legado de discriminação racial brasileiro, principalmente se ganham menos de 03 salários mínimos por mês, os quais, assim como aqueles com menor escolaridade, também relatam maior discriminação no atendimento de saúde.

\section{Colaboradores}

JA Chehuen Neto foi o orientador do grupo, responsável pela revisão e aprimoramento de todas as etapas da pesquisa, desde a definição do tema à submissão final do trabalho, aprofundamento da discussão e lapidação do artigo como um todo. GM Fonseca auxiliou na preparação do Protocolo de Pesquisa, construção do instrumento de coleta de dados e do TCLE, participação na redação da introdução, discussão, conclusão e resumo. IV Brum, JLCT Santos e TCGF Rodrigues participaram da revisão da literatura, preparação do Protocolo de Pesquisa, construção do instrumento de coleta de dados e do TCLE, aplicação do instrumento de coleta de dados, tabulação dos dados, elaboração da introdução, discussão, conclusão e resumo. KR Paulino colaborou na revisão da literatura, preparação do Protocolo de Pesquisa, construção do instrumento de coleta de dados e do TCLE, tabulação dos dados, elaboração da introdução, discussão, conclusão e resumo. RE Ferreira trabalhou na elaboração da metodologia e dos resultados, construção das tabelas de resultados, análise estatística/cruzamento de dados. 


\section{Referências}

1. Brasil. Ministério da Saúde (MS). Política Nacional de Saúde Integral da População Negra. Brasília: MS; 2007.

2. Brasil. Ministério da Saúde (MS). Secretaria de Gestão Estratégica e Participativa. Departamento de Apoio à Gestão Participativa. Políticas de promoção da equidade em saúde. Brasília: MS; 2013.

3. Maio MC, Monteiro S. Cidadania e saúde: etnicidade raça e saúde no Brasil: questões e desafios. Rio de Janeiro: Editora Fiocruz; 2005.

4. Brasil SA. A política de saúde da população negra do brasil: atores políticos, aspectos étnicos-raciais e principais tensões do campo [dissertação]. Salvador: Universidade Federal da Bahia; 2011.

5. Instituto Brasileiro de Geografia e Estatística (IBGE) Censo Demográfico 2010. Rio de Janeiro: IBGE; 2011.

6. Instituto de Pesquisas Econômicas Aplicadas (IPEA) Desigualdades raciais no Brasil: um balanço da intervenção governamental. Brasília: IPEA; 2002

7. Pinto EA, Souza R. Etnicidade e saúde da população negra no Brasil. Cad Saude Publica 2002; 18(5):11441145 .

8. Bastos JL, Peres MA, Peres KG, Dumith SC, Gigante DP. Diferenças socioeconômicas entre autoclassificação e heteroclassificação de cor/raça. Rev Saude Publica 2008; 42(2):324-334

9. Paixão M, Rossetto I, Montovanele F, Carvano LM. Relatório Anual das Desigualdades Raciais no Brasil; 2009. 2010. Rio de Janeiro: Garamond; 2010.

10. Freyre G. Casa Grande e Senzala, Rio de Janeiro: Liv. José Olympio; 1975.

11. Holanda SB. Raízes do Brasil. 26a ed. São Paulo: Companhia das Letras; 1995.

12. Chehuen Neto JA, Lima WG. Pesquisa Quantitativa. In Chehuen Neto JA, organizador. Metodologia da Pesquisa Científica: da graduação à pós-graduação. Curitiba: CRV; 2012. p. 147-154.
13. Brasil. Ministério da Saúde (MS). Conselho Nacional de Saúde. Resolução n 466, de 12 de dezembro de 2012. Diário Oficial da União 2013; 13 jun.

14. Moiomaz SAS. Satisfação e percepção do usuário do SUS sobre o serviço público de saúde. Physis 2010; 20(4):1419-1440.

15. Kalckmann S, Santos CGD, Batista LE, Cruz VM. Racismo institucional: um desafio para a equidade no SUS. Saúde soc. 2007; 16(2):146-155.

16. Fundação Perseu Abramo (FPA). Discriminação raciale preconceito de cor no Brasil. São Paulo: FPA; 2003.

17. Brasil. Ministério da Saúde (MS). Manual de Doenças mais Importantes, por Razões Étnicas, na População Brasileira Afro-descendente. Brasília: MS; 2001.

18. Lima M. Desigualdades raciais e políticas públicas: ações afirmativas no governo Lula. Novos estud. - CEBRAP 2010; 87:77-95.

19. Instituto de Pesquisa Econômica Aplicada (Ipea). Nota Técnica, Vidas Perdidas e Racismo no Brasil. Brasília: IPEA; 2013.

20. Chor D, Lima CRA. Aspectos epidemiológicos das desigualdades raciais em saúde no Brasil. Cad Saude Publica 2005; 21(5):1586-1594.

21. Santos JE, Giovanna CS. Narrativas dos profissionais da atenção primária sobre a política nacional de saúde integral da população negra. Saúde debate 2013; 37(99):563-570.

Artigo apresentado em 30/08/2014

Aprovado em 05/12/2014

Versão final apresentada em 07/12/2014 